\title{
La parentalité : où se situe l'autonomie des parents ?
}

Fabienne Berton

\section{Diversification des configurations familiales et transformation de l'action publique}

Le modèle traditionnel de la famille dans le cadre du mariage a laissé la place à d'autres modèles. En 2011, $55 \%$ des enfants naissent hors mariage (et $65 \%$ des premiers nés) contre $6 \%$ en $1970,18 \%$ des enfants vivent au sein de familles monoparentales et $11 \%$ dans une famille recomposée ${ }^{1}$. L'accroissement du nombre de séparation des couples parentaux avec son corollaire l'augmentation des familles monoparentales et des familles recomposées, les pratiques de procréation médicalement assistée (PMA) à l'étranger des femmes homosexuelles ${ }^{2}$ et la réflexion sur l'ouverture de la PMA à toutes les femmes ont élargi les repères et les normes familiales et parentales.

Face à ces reconfigurations de la famille contemporaine, on assiste, selon Gérard Neyrand (2011), à une nouvelle régulation publique du domaine privé qui prend pour objet le lien parental et non plus la famille elle-même, ni le couple parental: "l'enfant fait famille». La culture de l'enfance constitue la base de la valorisation de la relation parentale. Mais les parents d'aujourd'hui, confrontés à une exigence d'autonomie qui s'applique à tous, y compris aux enfants qui n'en ont pas encore les moyens, se trouveraient, selon Marcel Gauchet (2007), souvent désemparés, oscillant entre hyper-protection et abstention. Face à ce constat, les professionnels de la petite enfance se donnent et reçoivent pour mission d'activer et de soutenir les fonctions parentales. Le modèle de l'activation se décline aussi dans les politiques familiales qui engagent les parents dans des contrats de responsabilité : livret de paternité, contrat de réussite éducative, ... Ce devoir d'engagement a renforcé l'exigence faite aux parents, notamment les plus vulnérables socialement, de mener un travail autour de leur parentalité. Les dispositifs d'aide à la parentalité se sont multipliés, et les familles jugées «à risque » ont été fortement encouragées à s'y intégrer. Dans ce contexte, la question explorée par ce chapitre est celle des figures d'autonomie parentale repérables dans les interactions entre les familles et les professionnels de la petite enfance à l'occasion de la naissance d'un bébé. Nous partons d'une définition de l'autonomie située : «L'actuel contrat social semble encourager l'autonomie de chacun des membres de la société pour le bien commun : est autonome socialement une personne "productive", qui apporte une valeur ajoutée à l'effort national et ne s'inscrit pas dans un schéma "assistantiel" » [Chaffaut et al., 2003]. Une première partie précise l'esprit des politiques de la parentalité. Une seconde présente la politique en actes et en particulier les moyens dont se dotent les professionnels pour conduire les parents dans la voie de cette autonomie située. Une dernière partie expose quatre figures d'interactions, correspondant à différentes conceptions de l'autonomie qui synthétisent les situations parentales et dont certaines s'écartent de l'autonomie située voulue par la politique menée.

\section{« Etre parent face aux institutions »}

\section{Les interactions entre les familles et les professionnels de la petite enfance à l'occasion de la naissance d'un bébé}

Le laboratoire LISE CNRS, CNAM UMR3320 a mené entre 2012 et 2014, dans le cadre d'une convention de recherche signée avec la CNAF (appel à projet de recherche 2011 «Parentalité(s), production et réception des normes »), un travail sur les normes de parentalité à l'œuvre dans les interactions entre les familles et les professionnels sociaux et médico-sociaux [Berton et al., 2015a et b]. L'objectif de notre recherche est de mettre au jour, à partir du discours des parents, les divergences et les conflits qui se manifestent dans les rapports entre familles et les professionnels autour des normes éducatives. Nous avons interrogé une soixantaine de familles sélectionnées par niveau de revenu, nombre d'enfants et localisation géographique (la Côte-d'Or et la Seine-Saint-Denis) qui ont pour point commun la naissance

\footnotetext{
${ }^{1}$ Source : Insee, Statistiques d'état civil sur les naissances, France métropolitaine.

${ }^{2}$ La PMA est en France strictement réservée aux couples hétérosexuels inféconds.
} 
d'un premier ou d'un nouvel enfant en 20113. Quatre catégories de familles, hors de la norme de la famille nucléaire de deux enfants ont été interrogées : les familles nombreuses, plus de trois enfants ; les familles d'origine étrangère ; les familles recomposées et les familles homoparentales. En prolongement des familles, nous avons rencontré un peu plus d'une vingtaine de professionnels avec lesquels les familles sont effectivement en contact dans les deux premières années de leur bébé : médecins, sages-femmes, assistantes maternelles, psychologues, assistantes sociales, infirmières, puéricultrices, agents administratifs travaillant dans des structures telles que les maternités, la Protection Maternelle et Infantile (PMI), les crèches, les structures multi-accueil (structures mixtes qui regroupent une crèche et une halte-garderie), les Relais d'assistantes maternelles (RAM, lieux d'information, de rencontre et d'échange au service des parents, des assistantes maternelles et des professionnels de la petite enfance), les lieux d'accueil enfantsparents (ouverts aux enfants âgés de moins de six ans, accompagnés de leur(s) parent(s) ou d'un adulte familier pour participer à des temps conviviaux de jeux et d'échanges.), les centres sociaux, les associations d'aide aux parents, la caisse d'allocations familiales, le bureau d'aide sociale...

\section{Les politiques de soutien à la parentalité}

Mary Daly, féministe et philosophe, propose la définition suivante du soutien à la parentalité : «Un ensemble de services et de mesures organisés cherchant à avoir une incidence sur la manière dont les parents exercent leur rôle de parent en leur donnant accès à une gamme de ressources utiles pour augmenter leur compétence en matière d'éducation des enfants »[Daly, 2013, p. 162, cité par Claude Martin, 2014, p. 11].

Les politiques de soutien à la parentalité s'inscrivent dans une nouvelle orientation des politiques sociales menée depuis les années 1990, qualifiée d'investissement social car les politiques sont conçues comme un facteur productif susceptible de rendements. Soutenus par les institutions européennes, la CEE (Communauté Économique Européenne) et internationales, l'OCDE (Organisation de Coopération et de Développement Économiques), les investissements sociaux consistent à investir dans les personnes en adoptant des mesures pour renforcer leurs compétences et leurs capacités et leur permettre de participer pleinement au marché du travail et à la société4. Largement inspirée des travaux d'Anthony Giddens, de Gøsta Esping-Andersen et du prix Nobel d'économie, James Heckman (2000), une telle politique ressort non seulement du libéralisme en permettant l'accès des individus aux biens et services marchands, mais aussi du développement des capacités des personnes : leur capabilité, leur pouvoir d'agir, leur empowerment ${ }^{5}$ selon les principes d'Amartya Sen et de la théorie du capital humain de Theodore Shultz. L'idée de base en matière de parentalité est que le succès de l'enfant est davantage garanti par le soutien qu'il reçoit de ses parents, bien avant celui de la collectivité, d'où l'intérêt de prendre pour cible les pratiques éducatives et les comportements des parents : "Désormais, le parentage (parenting) n’est plus considéré simplement comme un lien personnel caractérisé par l'amour et le care. Au lieu de cela, il a été redéfini comme un travail nécessitant une expertise et des compétences particulières qui doivent être enseignées par des professionnels qualifiés » [Gillies, 2008, p. 95-96, cité par Claude Martin, 2014]. C’est en ce sens que Claude Martin parle de turning to parenting dans les politiques familiales à partir des années 1990.

\section{La politique de la parentalité en acte : les actions publiques en Seine-Saint-Denis et en Côte- d'Or}

Comment cette nouvelle orientation se décline-t-elle sur le terrain?

- $\quad$ Un premier échelon de mise en œuvre des politiques sociales en France est le niveau départemental. Les politiques sociales comptent au nombre des attributions des conseils départementaux. Deux départements aux caractéristiques sociodémographiques contrastées ont été enquêtés : la SeineSaint-Denis et la Côte d'Or ;

\footnotetext{
${ }^{3}$ Les familles qui avaient également des enfants plus âgés ont aussi abordé, dans leur entretien, d'autres âges de l'enfance, la scolarité en particulier.

${ }^{4}$ Commission Européenne, Pacte d'investissement social, 1er mars 2013.

${ }^{5}$ L'empowerment peut être défini comme le pouvoir d'agir d'individus ou de collectifs avec un objectif d'émancipation [Bacqué et Biewener, 2013]. Définition fournie par Claude Martin, 2014.
} 
Un deuxième échelon est l'action des professionnels de terrain sur le territoire départemental. Ces professionnels ont des champs d'intervention et de compétence divers en fonction de leur profession : sage-femme, assistante sociale, psychologue, médecin, assistante maternelle...

\subsection{Les actions publiques en lien avec la parentalité dans les deux départements investigués}

\section{La Seine-Saint-Denis développe les places d'accueil pour les tout-petits}

La Seine-Saint-Denis est un département marqué par un taux d'immigration très élevé, une proportion importante de familles pauvres et vulnérables. En dépit des efforts, les moyens financiers et humains ne sont pas à la hauteur des besoins.

La garde des enfants est le problème majeur rencontré par les parents. Seuls trois enfants de moins de trois ans sur dix ont un mode de garde contre plus de cinq sur dix au plan national. Si l'on tient compte de ses besoins, la Seine-Saint-Denis est largement déficitaire. Quel que soit le mode de garde considéré, l'offre dans le département est parmi les plus faibles d'Île-de-France, néanmoins l'accueil collectif est privilégié et son taux est supérieur à celui de France métropolitaine. La Caisse d'Allocation Familiale (CAF) de la Seine-Saint-Denis met en œuvre la création de nouvelles places d'accueil pour les tout-petits. Elle a engagé avec le Conseil général un schéma départemental de la petite enfance et de la parentalité qui cible les objectifs suivants :

- $\quad$ Réduire les inégalités territoriales en matière d'accueil collectif et individuel du jeune enfant ;

- Répondre aux besoins spécifiques des familles pour leurs enfants en situation de handicap ou pour les enfants de familles vulnérables ;

- $\quad$ Mailler le territoire en termes d'offre d'accompagnement à la parentalité ;

- $\quad$ Faciliter l'action coordonnée des acteurs sur le territoire ;

- Améliorer l'information des parents sur l'offre de service aux familles.

\section{La Côte-d'Or engage une politique active et innovante en faveur de l'accompagnement à la parentalité}

Dans un environnement rural, peu dense et vieillissant, le département de Côte-d'Or abrite la population la plus jeune et la plus diplômée de la région ; $69 \%$ de sa population vit en zone urbaine. La zone d'emploi de Dijon est la moins touchée du département par la crise.

Le schéma départemental Enfance - Famille de la Côte-d'Or ${ }^{6}$ infléchit les politiques publiques vers l'accompagnement à la parentalité. De nouvelles normes d'action publique se dessinent, mettant l'accent sur plusieurs priorités départementales :

- $\quad$ Faire évoluer les rapports des professionnels chargés de la petite enfance avec les parents. Les professionnels doivent se garder de toute position surplombante et axer l'accompagnement sur la valorisation des compétences parentales et non la correction de leurs incapacités ;

- Développer les structures d'accueil collectives et des lieux collectifs pour les assistantes maternelles, afin notamment de lutter contre les inégalités sociales ;

- $\quad$ Favoriser les lieux d'accueil/écoute parents-enfants et parents pour lutter contre l'isolement des familles et développer le lien social entre les familles et entre familles et professionnels dans une dynamique de coéducation ;

- $\quad$ Agir en direction des pères pour instituer les règles d'une coparentalité effective.

\subsection{Les actions des professionnels de terrain : coparentalité et coéducation}

Aux yeux des professionnels de la toute petite enfance, nombreux à être consultés à cet âge de la vie, les parents de tous milieux, aisés comme défavorisés, apparaissent, comme peu sûrs du bien-fondé de leur autorité et de leur capacité à éduquer leur enfant. Les professionnels énoncent un jugement général qui est loin de recouvrir la variété des situations sociales rencontrées dans notre enquête. Ce diagnostic de fragilité des parents sur lequel ils assoient leurs interventions est, selon eux, étayé par plusieurs facteurs :

- La prolifération des sources légitimes de normes. Séverine Gojart [2010] a montré, concernant l'allaitement, la concurrence des savoirs en présence (considérés comme innés, familiaux, médicaux,

\footnotetext{
${ }^{6}$ Schéma Départemental Enfance Famille de la Côte-d’Or, Schéma partenarial Etat - Conseil Général (2010-2014).
} 
psychologiques, vulgarisés par les médias...) diversement mobilisés et combinés selon les trajectoires et les positions sociales des familles: "Les parents sont de plus en plus perdus parce qu'ils entendent des choses partout. [...] Comment s'y retrouver là-dedans?" (Responsable d'une structure multiaccueil petite enfance en Côte-d'Or) ;

- Des fonctions parentales qui ne suffisent plus à porter seules l'autorité parentale : «Dans la fonction parentale, il y a quelque chose qui a tenu pendant des années voire des siècles et qui aujourd'bui ne tient plus [...] Avant on était parent, il fallait que ça file droit. » (Psychologue d'un lieu d'accueil enfants-parents en Côte-d'Or). La question de l'exercice de l'autorité des parents est un clivage important au sein des familles de notre échantillon où nous avons rencontré d'un côté des familles qui mettent l'accent sur l'autorité traditionnelle des parents et le savoir naturel de la mère et d'un autre des familles caractérisées par le désir d'être de bons parents selon les normes actuelles, qui s'interrogent sur leurs pratiques parentales, recherchent les conseils et sont prêts à se remettre en cause [Berton et al., 2015b] ;

- L'angoisse parentale de ne "pas être à la hauteur»: une culpabilité parentale déstabilisante pour l'enfant: "Il y a une espèce de culpabilisation des parents dès que l'enfant montre des signes qu'il ne va pas. [...] Le parent va tout de suite se culpabiliser. Ca a un impact sur l'enfant qui se demande qui tient le gouvernail. » (Psychologue d'un lieu d'accueil enfants-parents en Côte-d'Or)

Les questions de l'autorité, des limites à fixer aux enfants et du désarroi, voire de l'angoisse des parents par rapport à leur rôle ressortent assez systématiquement du discours des professionnels rencontrés. Pour comprendre ces discours des professionnels de la toute petite enfance, il faut se référer à différents travaux qui font état de la construction du jugement des professionnels vis-à-vis des parents en fonction de normes de genre et de classe [Serre, 1998 ; Gojard, 2010 ; Camus et Oria, 2011]. Ces travaux révèlent l'existence de normes dominantes portées par les institutions ou le discours public qui rencontrent des styles parentaux socialement distribués, qui s'en éloignent plus ou moins. La distance sociale entre le professionnel et la famille donne à voir des jugements, des interactions et des façons de faire différenciées. La question de la «perte de repères» des parents par rapport à l'éducation de leurs enfants qui ressort souvent du discours des professionnels prend une autre dimension à l'aune de ces travaux sociologiques.

Une chargée de mission parentalité au ministère de l'Intérieur s'accorde avec deux responsables de lieux d'accueil pour penser que les parents, ceux notamment immigrés et/ou de milieu défavorisé, sont surtout angoissés, perdus, et sans repères face à l'éducation de leurs enfants reprenant en cela le discours misérabiliste sur les classes populaires complètement dominées. Elle s'insurge néanmoins contre l'idée de parents démissionnaires :

"Des études ont montrê $[. .$.$] que les parents, y compris immigrés, ne sont pas du tout démissionnaires, qu'ils$ aimeraient bien faire, qu'ils sont conscients que c'est difficile. Ils sont très intéressés par la réussite scolaire et professionnelle de leurs enfants. ». (Chargée de mission du Bureau de l'intégration territoriale au ministère de l'Intérieur)

Ce fait est confirmé par l'exploitation de la cohorte $\mathrm{ELFE}^{8}$ où aux deux mois du bébé, les familles de plus de 3 enfants et celles originaires du Maghreb et d'Afrique de l'Ouest sont dans une proportion plus importante que l'ensemble de la cohorte à souhaiter à leur bébé la réussite sociale et un travail intéressant (versus un épanouissement personnel et la construction d'un réseau relationnel) qui peuvent être interprétés en référence à leur situation socioéconomique et/ou à leur volonté d'intégration dans le pays [Berton et al., 2015a].

Les doutes des professionnels sont renforcés par l'isolement des parents. Les professionnels rencontrent davantage de femmes seules, de mères jeunes, de séparations précoces et d'absence de famille élargie. C’est

${ }^{7}$ L'interlocuteur fait probablement référence à Lahire (1995), mais aussi à d'autres travaux plus récents rappelés dans Brinbaum (2013) concernant plus spécifiquement les familles maghrébines. Ces travaux mettent en avant les aspirations fortes des familles immigrées vis-à-vis de l'école, celle-ci étant perçue comme un moyen d'intégration, de mobilité sociale et d'anticipation des difficultés et des discriminations rencontrées sur le marché du travail.

${ }^{8}$ ELFE: Étude longitudinale française depuis l'enfance (www.elfe-france.fr) constituée en unité mixte INED-INSERMÉtablissement Français du Sang (EFS) et financée au titre des équipements d'excellence (2e appel à projet), PROJET RE-CONAI : Plateforme de Recherche sur les Cohortes d'enfants suivis depuis la NAIssance. 18000 enfants nés en France métropolitaine en 2011 sont suivis du point de vue de la santé et de toutes les composantes des sciences sociales de leur naissance à leurs vingt ans. 
le défaut de lien social et le déficit d'assurance sur le rôle de parent qui sont mis en avant. Pour lutter contre l'isolement des familles, les professionnels les incitent à recourir aux services proposés.

"Dans les salles d'attente en PMI, on a mis en place des psychomotriciennes, des éducatrices de jeunes enfants, des infirmières qui peuvent faire circuler la parole et éviter qu'il y ait des choses trop grosses qui se passent. » (Médecin de Protection Maternelle et Infantile en Côte-d'Or).

Les relations avec des familles en grande difficulté posent des interrogations sur les priorités : comment accompagner au mieux sans décharger les parents de leur rôle ? La responsable d'une structure d'accueil de Côte-d'Or explique son hésitation face aux demandes de garde à temps complet faites par des mères qui recherchent du travail. Ils insistent également sur l'importance de réaffirmer la place des pères. Le développement de la coparentalité constitue pour les professionnels un enjeu essentiel. Dans un centre social, le rôle du père est ainsi systématiquement valorisé auprès des jeunes mères séparées :

«On parle beaucoup du lien avec l'autre parent, qui souvent n'est pas là. Mon objectif c'est de faire réfléchir cette maman sur la place qu'elle veut et qu'elle peut donner à ce père. » (Assistante sociale d'un Centre social en Côte-d'Or).

Notion plus large que la coparentalité, la coéducation est encouragée. Elle se manifeste par l'utilisation d'un vocabulaire nouveau. Les professionnels parlent de "compétences parentales », d'accompagnement à la parentalité ». Utiliser la notion de «travail parental», comme le proposent Anne Verjus et Marie Vogel (2009), permet de tenir compte de l'apprentissage nécessaire au " devenir parents ", de renvoyer à l'ensemble des tâches matérielles de prise en charge et de soins aux enfants mais également aux aspects symboliques, cognitifs et affectifs, de la parentalité.

«Le soutien à la parentalité, pour moi, c'est comment chaque professionnel, dans sa fonction autour de la naissance, peut donner une attention qui permette aux parents d'être suffisamment rassurés [...] pour powvoir, eux-mêmes, se préoccuper de leur enfant. » (Psychologue2 d'un hôpital public de banlieue populaire)

La coéducation se manifeste par la volonté d'estomper les barrières entre les professionnels et les parents, « tous acteurs ensemble ». Elle cherche à casser l'asymétrie des relations entre professionnels et usagers, à impliquer ces derniers dans les actions d'accompagnement proposées et à promouvoir les «savoirs du proche ». On retrouve cette volonté de créer une dynamique collective dans un Lieu d'Accueil EnfantsParents (LAEP), ou dans un Relais d'Assistantes Maternelles (RAM) en Côte-d'Or, même s'il existe des résistances à ce mouvement de la part des assistantes maternelles. Pour mettre en œuvre le principe de coéducation avec les parents, les professionnels rappellent qu'il « n'existe pas de définition unique de la bonne parentalité ». Ils font le pari de la socialisation entre pairs comme facteur d'autonomie des parents, par les rencontres dans des espaces de convivialité comme les LAEP. Ces lieux peuvent être considérés comme l'archétype de la parentalité réflexive.

«On ne fonctionne pas selon le principe des activités, mais selon l'ambiance. On travaille sur l'instant, la vitalité du lien au moment où il est. » (Psychologue d'une Maison d'accueil en Côte-d'Or).

Deux principes sont énoncés : ne plus partir des limites des parents mais de leurs compétences et s'appuyer sur le collectif pour permettre aux parents d'échanger sur leurs pratiques éducatives. Le travail autour des compétences parentales s'inscrit dans des pratiques très concrètes, qui associent les parents au travail éducatif auprès des enfants :

«Par exemple, l'action " jeux de mots pour grandir » travaille vraiment sur les compétences parentales. [...] On a choisi de faire des ateliers parents/enfants autour de la question du vocabulaire. Une professionnelle de la petite enfance avec l'assistante sociale, une fois par semaine, accueillent 4 enfants et leurs parents. [...] L'idée c'est que les mamans fassent reprendre leurs enfants [à la maison]. Les parents ont cette compétence-là. Il ne faut pas que les professionnels leur enlèvent.» (Directrice d'un centre social en Côte-d'Or).

Dans cette voie participative, les échanges entre parents deviennent des appuis essentiels à l'accompagnement à la parentalité. Favoriser le dialogue entre parents permet de restaurer leur « pouvoir 
d'agir » et leur confiance en eux. Les structures multiplient les espaces de rencontre, tels que ce caféparents mis en place dans un centre multi-accueil ou cet espace de jeux où sont conviées les familles dans un centre social. Un centre social de Côte-d'Or montre l'intérêt d'un groupe de parole organisé tous les trimestres avec les familles et un psychothérapeute. Le groupe a lieu le samedi, jour choisi pour impliquer les pères. Le collectif est considéré comme un levier important pour instaurer la confiance et les parents " travaillent sur leur parentalité sans en avoir l'air » (Directrice d'un centre social en Côte-d'Or). Il s'agit alors de « ne pas juger », de respecter la diversité des modèles. Les professionnels s'efforcent, notamment face aux familles immigrées, de concilier le respect des pratiques éducatives avec les codes du pays d'accueil. Les structures d'accueil font preuve d'une relative souplesse sur les horaires des femmes africaines, sur les différentes habitudes culturelles en termes de sommeil, d'alimentation ou d'autorité. L'absence de jugement sur la diversité culturelle fait partie des normes partagées.

\section{Quatre figures de l'autonomie parentale}

Face à ces actions, quatre figures principales d'autonomie parentale apparaissent qui montrent :

- L'autonomie encadrée des familles qui s'insèrent dans cette nouvelle orientation de la politique familiale et qui jouent le jeu de la réflexivité sur leurs pratiques quotidiennes ;

- L'autonomie contrainte ou la dépendance subie des familles auxquelles les nouvelles orientations de la politique familiale et sa conception de l'autonomie sont d'autant plus imposées qu'elles se trouvent dans une situation sociale qui les met sous la coupe des professionnels ;

- La non autonomie ou la dépendance choisie des familles qui recherchent avant tout une protection de la part des professionnels ;

- $\quad$ L'autonomie complète9 des familles qui :

$\checkmark \quad$ Pour certaines sont laissées dans l'abandon de la part des professionnels du fait de l'urgence sociale ;

$\checkmark \quad$ Pour d'autres, ne souhaitent pas, pour diverses raisons, recourir aux différents services de l'aide sociale (non recours) ;

$\checkmark \quad$ Pour d'autres encore, rejettent les nouvelles orientations de la politique familiale par des actes de résistance ouverte, ou les mettent à distance par des contournements discrets.

\subsection{L'autonomie encadrée}

Les professionnels adressent aux parents une invitation à être de "bons parents " ce qui signifie en particulier être capables de réflexivité sur leurs pratiques et prêts à coopérer avec les professionnels de la petite enfance. Les familles qui acceptent de partager leurs expériences et de participer aux différents groupes et rencontres mis en place par les professionnels, entrent dans un cadre qui est mis à leur disposition. Lorsque les parents acceptent de coopérer pleinement avec les professionnels de la petite enfance pour les soins et l'éducation donnés à leurs enfants, il y a congruence entre les normes véhiculées par les professionnels et celles des parents, si bien que l'on rencontre principalement ces situations dans les catégories sociales moyennes ou aisées. Il n'est pas surprenant de rencontrer ces formes de coopération harmonieuse avec les professionnels dans les rapports entre parents et structures socio-éducatives par exemple.

Perçues la plupart du temps très positivement, les structures d'accueil sont reconnues généralement pour la qualité de leur service : éveil de l'enfant, professionnels formés, période d'adaptation utile et bien menée, écoute des parents et des enfants, espace rassurant. Cette mère de milieu aisé, vivant dans une grande agglomération, insiste sur la qualité des équipes professionnelles, compétentes et ouvertes vis-à-vis des parents :

"Les personnes qui travaillent ont vraiment une belle approche de la séparation de l'enfant. Même si l'adaptation peut être contraignante, je me dis que c'est une bonne chose pour l'enfant. Je trowve que c'est bien fait aujourd'bui. » (Mère de famille nombreuse d'un milieu aisé).

\footnotetext{
9 Autonomie complète en ce sens qu'elle correspond à la définition première de l'autonomie : le droit pour un individu de déterminer librement les règles auxquelles il se soumet.
} 
Accoutumées à la réflexivité sur leur rôle de parent en raison de leur expérience spécifique, les familles homoparentales entrent aussi aisément dans des relations de coopération harmonieuse avec les structures d'accueil de la petite enfance, à condition que la crainte de stigmatisation soit écartée. Certains homoparents précisent leur attachement particulier à cette dimension collective, qui favorise une intégration jugée compromise par leur spécificité familiale. Un couple de femmes parisiennes affiche clairement les enjeux de normalisation que recouvrait pour elles l'inscription en structure collective : «On voulait une structure collective. C'est important de s'investir, de s'intégrer pour passer "inaperçues", ce n'est pas le terme mais pour être considérées comme une famille "normale". C'est important, pour nous, de ne pas marquer encore plus notre différence, si on veut être intégrées. »

\subsection{La dépendance subie}

Dans les situations de grande précarité sociale, le caractère intrusif de l'institution peut être ressenti violemment, en particulier dans les cas de placement d'enfant. Les interventions socioéducatives peuvent être très mal perçues. Comme c'est le cas dans cette famille dont le fils aîné, issu d'une union précédente et suite au constat de maltraitance, est accueilli en foyer éducatif durant la semaine. Toute la fratrie est suivie en milieu ouvert. Cette mesure est incomprise des parents qui n'ont pas le sentiment d'être aidés, bien au contraire : "Ce n'est pas une aide du tout, ça enfonce plus les gens même! "Ils la vivent comme un acharnement des services sociaux ${ }^{10}$. Les autres enfants de la famille ont également un suivi socio-éducatif, en raison, selon les parents, de la prise en charge de l'ainé. Les parents expriment leur impuissance vis-àvis de l'intervention des services, ce qui les conduit à rejeter toute autre aide. Ils ne savent pas, par exemple, s'il y a des maisons de quartiers ou des associations autour de leur domicile, mais de toute façon ils ne souhaitent pas y faire appel car «Ils ne servent à rien, ce n'est pas la peine ».

La dépendance subie aux professionnels peut aussi prendre la forme de l'imposition d'un mode de vie non souhaité, pour les familles monoparentales plus particulièrement. Aux yeux des professionnels rencontrés, l'absence du père fait soupçonner des difficultés. Cette situation est d'emblée jugée grave du fait de ses répercussions sur l'enfant qui n'aurait plus de limites. Ils considèrent donc qu'il est de la responsabilité de la mère de ne pas couper le lien avec le père. Pourtant, de nombreuses mères, séparées ou non, affirment qu'elles sont la seule représentante de l'autorité dans la famille, le père étant peu présent. Dans un cas extrême, c'est la mère qui, seule, assume tout. Marcel Gauchet affirme qu': "Il n'est pas excessif de dire que l'évolution va vers un type de familles où les mères sont en même temps les pères tandis que le géniteur n’a qu'une existence résiduelle. » [Gauchet, 2007 :17]. De nombreuses mères revendiquent ce rôle

«Je suis la responsable d'une famille et je suis la maman, je suis le papa, je suis tout. [...] Je me gère moi toute seule et je tiens debout pour tout le monde. » (Mère au chômage de milieu populaire, seule avec 4 enfants).

"Quand S. est né, il s'en est quand même un petit peu occupé, mais il ne fallait pas que ce soit trop non plus. Pour lui, il ne voulait pas être un papa à la maison. Même si c'est lui qui ne travaillait pas et moi qui travaillais. » (Mère d'une famille monoparentale de milieu populaire, le père est parti aux trois mois du le bébé)

\subsection{La dépendance choisie}

Devant la multiplication des difficultés auxquelles elles doivent faire face, certaines familles demandent que les professionnels les aident concrètement et les protègent. On est alors à l'opposé de l'autonomie, et dans une situation bien plus proche des modalités traditionnelles d'intervention de l'aide sociale. Il s'agit de répondre à une demande de dépendance qui n'est pas vécue par les familles sur le mode de la privation de liberté mais sur celui du soutien effectif et nécessaire. Certains professionnels répondent à cette demande, quitte à outrepasser parfois les limites de leur champ d'intervention. Plusieurs mères d'origine étrangère ont ainsi expliqué comment les services sociaux ont pu jouer un rôle de rempart institutionnel et humain, en particulier dans des situations de violence conjugale ou de propriétaire malveillant. Une mère d'origine marocaine, divorcée et seule avec quatre enfants confie ainsi : "Ils m'ont expliqué la loi, ils m'ont sauvée et ils ont sauvé mes enfants aussi $»$. Dans une autre situation des professionnels de la CAF et de la PMI se sont engagés aux côtés de cette mère de famille nombreuse : "C'est eux qui ont été debout avec moi. Tous m'ont soutenue, autant qu'ils sont». Pour elle, la PMI est devenue un second foyer.

${ }^{10}$ Cf. le chapitre d'Abdia Touahria-Gaillard dans cet ouvrage. 


\subsection{L'autonomie complète à l'égard de l'action des professionnels}

Que les familles soient abandonnées par les professionnels en raison du manque de moyens des services sociaux, qu'elles choisissent de ne pas recourir aux professionnels, qu'elles résistent plus ou moins vivement à leurs actions ou qu'elles les contournent et ne les utilisent que de façon délibérée, les familles sont, dans tous ces cas de figure, en situation de s'affranchir des professionnels et dans l'obligation trouver par elles-mêmes des ressources alternatives.

\section{L'abandon par les professionnels}

Les situations d'urgence laissent de nombreuses familles sans protection. Certains professionnels de SeineSaint-Denis, confrontés quotidiennement à des situations de détresse sociale et au manque de moyens pour y remédier, tentent surtout de parer à l'urgence en limitant leurs ambitions socioéducatives. Ils optent le plus souvent pour le respect des pratiques parentales, à partir du moment où la santé de l'enfant n'est pas en danger. Sur le plan éducatif, les professionnels pointent la difficulté d'identifier correctement des situations de danger dans un contexte où " les seuils de tolérance sont très élevés » (secrétaire d'accueil d'un Bureau d'Aide Sociale). Ils insistent sur la nécessité d'un apprentissage culturel pour comprendre correctement les fonctionnements familiaux méconnus comme par exemple, la spécificité du rapport à l'autorité ou du système d'entraide dans les familles africaines.

\section{Le non-recours aux professionnels}

Nous avons également rencontré des familles démunies qui n'ont pas recours à l'aide sociale par crainte. L'exemple d'une mère dont le mari est incarcéré illustre bien cette forme de non recours :

"Ily a les aides sociales, mais je ne peux pas vivre que d'aides sociales. [...]. Le père de mon fils, actuellement il ne peut pas m'aider. [...] On me dit qu'il va falloir écrire un courrier pour dire qu'il est emprisonné. J'ai peur que sa porte préjudice à notre histoire, le fait que je fasse une demande d'aide sociale. C'est comme si on mettait une plainte sur lui.» (Mère de famille nombreuse d'origine étrangère).

Une autre mère de famille nombreuse et recomposée en milieu populaire refuse d'aller à l'hôpital public. Ce refus se conjugue à un refus des modes de garde collectifs, argumenté par le manque d'attention et de soins dont les bébés seraient l'objet dans ces structures. Comme certaines autres familles, elle explique le non-recours à un mode de garde par sa conviction que la mère reste le meilleur système de garde. Elle est très fière d'annoncer que : «Mes enfants n'ont jamais été gardés.»

\section{Les résistances à l'action des professionnels}

C'est le plus souvent au nom de la défense et de l'affirmation de leurs propres valeurs que certaines familles résistent aux actions des professionnels ou les contournent. Leur autonomie n'est alors ni encadrée, ni contrainte, elle est complète en ce sens que les familles mènent leur vie selon leurs propres règles dictées par les valeurs qui sont les leurs. Il ressort des entretiens avec certaines familles nombreuses et/ou recomposées (particulièrement celles de milieu populaire ou d'origine étrangère et les familles nombreuses catholiques pratiquantes), la tonalité générale d'une mise à distance des institutions, perçues comme utiles mais dont il convient de contrôler l'intervention dans les familles. Cette distance découle d'une naturalisation de l'expérience parentale, qui va parfois conduire à des rapports de concurrence avec certaines institutions, notamment pour les couples disposant d'un schéma éducatif solide.

La contestation des pratiques éducatives des professionnels, peut conduire à retirer les enfants des structures publiques. Une mère catholique de Côte-d'Or estime ainsi : «Ils ont vraiment décidé que l'Etat laï devait se charger de l'éducation de nos enfants de $A$ à $Z$ et nous, parents, on n'a plus notre mot à dire $»^{11}$. Cette question de la concurrence éducative entre les parents et les instances de socialisation, l'école en particulier porte sur la charte de droits de l'enfant ${ }^{12}$, la nourriture dans les cantines, l'intervention de l'Etat dans les programmes scolaires... qui limitent le pouvoir parental. Le conflit se fixe sur des apprentissages jugés inadaptés au milieu scolaire ou trop choquant ("La théorie du genre», par exemple) ou qui incitent un enfant à faire appel à la police ou à l'assistante sociale. Cette mère, assistante maternelle d'origine algérienne, met en cause la façon dont l'école avertit les enfants de leurs droits dès le plus jeune âge et les encourage à

\footnotetext{
11 Il faut préciser que notre enquête dans les familles s'est principalement déroulée durant l'hiver 2013, au moment des débats parlementaires de la loi sur le mariage pour tous et des manifestations dites « Manif pour tous ».

${ }^{12}$ Charte qui, conceptuellement, est autre chose que la culture de l'enfant-roi décriée par certains professionnels et certaines familles.
} 
renverser l'autorité des parents : «La première année, quand ma fille est partie à l'école, elle est rentrée avec des paroles bizarres: "Maman, j'appelle la police, j'ai le droit de faire ça!"»».

Certains parents ont le sentiment de ne pas pouvoir exercer leur autorité comme ils l'entendent. La culture de l'enfance [Segalen, 2010] ${ }^{13}$ est incomprise par les familles assises sur une autorité traditionnelle selon un modèle issu de leur propre héritage familial, ce qui les incite à prendre des distances avec les professionnels et les institutions : «Des professionnels, destructurants, destructeurs, déstructurés 》 (mère de famille nombreuse). Ces familles se situent d'emblée hors de la norme commune en affirmant leur norme d'autorité traditionnelle et le savoir naturel de la mère. La prohibition des châtiments corporels est un autre point de tension. Certaines familles passent outre, elles ne prennent pas cette interdiction à la lettre. D'autres familles ressentent très fortement cette interdiction - d'autant plus fortement qu'elles sont en situation sociale précaire, souvent nombreuses et d'origine étrangère, et qu'elles sont «observées » en quasi permanence par les travailleurs sociaux - mais elles ne savent pas faire autrement : «I ci il faut parler, il ne faut pas taper, il faut parler, mais il faut parler jusqu'à quand? " (Mère de famille nombreuse). Mais d'un autre côté, certains professionnels font part d'un contexte général d'insuffisance d'autorité des parents : « Si les parents n'assurent plus, il va falloir créer des brigades de soutien à la parentalité. Vous voyez? Je plaisante mais c'est vrai qu'à un moment, il va falloir corriger.» (Sage-femme d'un hôpital public).

\section{Les contournements discrets}

Ainsi, des mères de familles nombreuses, des mères d'origine étrangère ou des mères qui exercent le métier d'assistante maternelle recourent de façon contrôlée et sélective aux institutions, font le tri dans les conseils, prescriptions, voire injonctions, adressés par les professionnels. Dans ce cas, leur expérience, acquise ou transmise, leur confère une certaine assurance face aux savoirs experts, y compris le pouvoir médical. Telle mère de famille nombreuse explique ainsi sur un mode ironique : «Avec l'expérience que j'ai, je dicte les ordonnances au médecin ». Des familles d'origine étrangère expliquent comment elles composent avec les professionnels, choisissant « d'en prendre et d'en laisser», de façon à préserver des savoir-faire traditionnels sans pour autant rejeter les conseils qu'on leur donne. Telle cette mère d'origine sénégalaise : "La culture francaise a des principes de développement de l'enfant. En même temps, on intègre aussi ce qu'on mange nous, dans nos babitudes. C'est pour ça que je dis : "c'est un mélange entre la culture européenne et notre culture "». D'autres mères préfèrent le contournement, tout en évitant le conflit : «Je dis oui et je fais à ma façon».

Le contournement peut être plus discret et exprimé de la part de familles largement insérées dans le maillage des aides sociales. Cette mère qui élève seule son bébé et bénéficie de nombreuses aides sociales est bien décidée à faire la part des choses des conseils d'éducation qui lui sont prodigués. L'expérience pratique qu'elle acquiert lui permet de s'autonomiser des prescripteurs [Gojard, 2010] :

«Comme certaines personnes qui disaient : "Laissez-le pleurer votre enfant! Arrêtez de l'allaiter toutes les $2 b^{1} / 2$ la nuit! Sinon, il ne fera jamais ses nuits! "... Des choses comme ça. Et finalement, je l'ai laissé pleurer de temps en temps et je me rends compte que ça ne me convenait pas. [...] Il faut se faire confiance. L'enfant est là, il a besoin de quoi ? De contact, un maximum. Ce n'est pas parce qu'il est tout le temps colle à nous sur notre dos en portage bébé que ça va être un enfant qui va être tout le temps collé à nous! Justement! Ils deviennent plus autonomes parce qu'ils ont eu toute la sécurité dont ils avaient besoin quand ils étaient petits... » (Mère d'une famille monoparentale de milieu populaire, le père est parti aux 3 mois du le bébé).

\section{Les ressources alternatives aux professionnels}

Alternativement aux actions des professionnels, certaines familles, les familles homoparentales et celles où la conviction religieuse prévaut, font largement appel à leur propre réseau : milieu associatif et groupes informels. Les familles homoparentales mobilisent un réseau associatif spécifique qui offre support et information sur les évolutions légales, écoute, conseils et un contexte ad hoc de socialisation pour leurs enfants : «Ca permet aussi de donner... pas des conseils mais plutôt de témoigner, d'échanger sur notre expérience, ce qu'on a vécu, ò̀ on a été. » Plusieurs parents font référence aux groupes de paroles qu'organisent ces associations: "Cela permet de se livrer et de partager ses doutes et d'avoir des réponses [...] cela donne des pistes. »

Les familles catholiques rencontrées mobilisent nombre de structures religieuses et éducatives, groupes de prière et réseaux d'entraide informels. Ces groupes constituent un soutien qui conforte les familles dans leurs choix éducatifs et religieux: "J'ai pris conscience que c'était une richesse » (Mère de famille nombreuse,

13 Martine Ségalen (2010) souligne le passage de l'adultocentrisme au pédocentrisme, tendance tournée vers la protection et l'affirmation des droits de l'enfant. Cette tension permanente entre "protection » et "libération» caractérise la spécificité de l'individualisation de l'enfant dans les sociétés contemporaines [de Singly, 2006]. 
milieu aisé). Ces groupes permettent aussi aux mères qui restent au foyer de conserver un réseau social: "Je trouve qu'il y a une véritable entraide entre mamans et il y a également beaucoup d'activités qui se créent entre les mamans ».

\section{Conclusion}

Dans un contexte de transformation des politiques de la famille dans le sens d'une logique d'accompagnement de la mission éducative des familles, les professionnels de la toute petite enfance développent des actions d'activation de la fonction parentale qui mobilisent principalement la réflexivité des parents, leur coopération, les échanges entre pairs et la coéducation. L'autonomie des parents est recherchée en ce sens qu'il ne s'agit pas d'agir à leur place mais de leur donner les moyens de mettre en œuvre leurs compétences parentales. Mais l'action des professionnels n'échappe pas aux caractéristiques des territoires et des populations auxquels elle se destine. Elle est partagée, selon les situations locales, entre accompagnement, soutien, contrôle et intervention d'urgence. Dans ce contexte et par rapport aux actions initiées par les professionnels, les figures de l'autonomie parentale sont multiples. Elles vont de l'autonomie encadrée en passant par l'autonomie contrainte, la dépendance subie, la dépendance choisie, pour arriver au non recours, à la résistance et au contournement plus ou moins forts. Finalement, l'autonomie cohérente avec la mise en œuvre de la politique de parentalité n'est qu'une forme d'autonomie parmi d'autres et les parents les plus autonomes sont ceux qui, pour diverses raisons, tiennent à distance les professionnels de la toute petite enfance. Bien qu'ils soient les plus autonomes, ces parents sont ceux qui n'entrent pas dans les mailles de la politique d'accompagnement à la parentalité prônée actuellement démontrant de ce fait la conception tout à fait particulière de l'autonomie qui y est incluse. La connaissance de ces diverses formes d'autonomie conduit à affirmer la nécessité d'une action publique plurielle. Sur le plan théorique, ce chapitre a présenté quelques illustrations du fait que l'autonomie est une notion située dans un système de dépendances sociales interactives où l'individuel et le social sont imbriqués. L'individu devient autonome en s'appropriant des normes et des règles trouvées dans le cadre de ses relations sociales et de sa vie en société.

\section{Bibliographie}

Bacqué M.-H., Biewener C. (2013), «L'empowerment, une pratique émancipatrice », Revue Projet n $336-$ 337, p. 186-187.

Berton F., de Bony J., Bureau M.-C., Jung C., Rist B., Touahria-Gaillard A. (2015a), «ÊEtre parent face aux institutions : normes de parentalité et injonctions paradoxales dans l'action publique », rapport final LISE CNRS/CNAM UMR3320 Convention CNAF/LISE CNRS, CNAM n 10/12, notifiée le 20 juillet 2012, janvier.

Berton F., de Bony J., Bureau M.-C., Jung C., Rist B., Touahria-Gaillard A. (2015b), «Être parent face aux institutions : normes, supports et résistances », Quatre Pages, Happy Hour du LISE, 9 juin.

Brinbaum Y. (2013), «Les familles immigrées et l'école: à l'encontre des idées reçues », Diversité, 4e trimestre, p. 152-163.

Camus J., Oria N. (2011), «Apprendre à être parent à la maternité : transmission et concurrence des savoirs ", Revue française de pédagogie, $\mathrm{n}^{\circ}$ 176, p. 73-82.

Chauffaut D., David E., avec la collaboration d'I. Aldeghi, V. Cohen-Solal, T. Mahé, M. Bouscasse (2003), «La notion d'autonomie dans le travail social, l'exemple du RMI», Crédoc, Cabier de recherche n 186, septembre.

Daly M. (2013), "Parenting support policies in Europe", Families, Relationships and Societies, vol. 2, n², p. $159-174$.

Gauchet M. (2007), «L'enfant du désir », Champ psy, n 47, p. 9-22. 
Gillies V. (2008), "Perspectives on Parenting Responsibility: Contextualizing Values and Practices", Journal of Law and Society, vol. 35, $\mathrm{n}^{\circ}$ 1, mars, 95-112.

Gojard S. (2010), Le métier de mère, Paris, La Dispute, coll. « Corps santé société ».

Hamel M.-P., Lemoine S., en collaboration avec C. Martin (2012), «Aider les parents à être parents. Le soutien à la parentalité, une perspective internationale», rapport du Centre d'analyse stratégique, septembre.

Lahire B. (1995), Tableaux de famille. Heurs et malheurs scolaires en milieux populaires, Paris, Seuil (éd. Points, 2012).

Martin C. (dir.) (2014), «Être un bon parent». Une injonction contemporaine, Rennes, Presses de l'EHESP, coll. «Lien social et politiques ».

Martin C. (2014), «Le soutien à la parentalité : une nouvelle politique en Europe ?», Production et réception des normes de « bonne » parentalité, Politiques sociales et familiales n 118, décembre, p. 9-22.

Neyrand G. (2011), Soutenir et contrôler les parents. Le dispositif de parentalité, Paris, Érès.

Segalen M. (2010), À qui appartiennent les enfants ?, Paris, Taillandier.

Serre D. (1998), «Le bébé "superbe" : la construction de la déviance corporelle par les professionnel(le)s de la petite enfance », Sociétés contemporaines n 31, p. 107-127.

Serre D. (2009), Les coulisses de l'État social. Enquête sur les signalements d'enfant en danger, Raisons d'agir, coll. «Cours et travaux».

Singly F. (de) (2006), Les adonaissants, Paris, Armand Colin.

Verjus A., Vogel M. (coord.) (2009), «Le travail parental : représentations et pratiques », Informations sociales, n 154, p. 4-6.

\section{Résumé}

Quelles figures de l'autonomie des parents observe-t-on à l'occasion de la naissance d'un bébé dans le cadre des politiques de la parentalité menées en France depuis les années 1990 ? S’il n’est pas explicitement question d'autonomie dans ces politiques, il est question de responsabilisation des parents. Celle-ci est fondée sur le déploiement de leur pouvoir d'agir à travers des actions qui visent à les aider et à les soutenir et donc à les guider vers une «bonne parentalité » qui leur reviendra d'engager de façon autonome. Mais l'action des professionnels de la toute petite enfance est partagée, selon les situations, entre accompagnement, soutien, contrôle et intervention d'urgence. Dans ce contexte, les figures de l'autonomie parentale à l'égard de ces professionnels sont multiples entre autonomie encadrée, autonomie contrainte, dépendance choisie, dépendance subie, non recours, résistance et contournement.

Mots-clé : naissance, politique sociale, famille, professionnels de la toute petite enfance, activation, autonomie, dépendance, résistance 